\title{
Design of Cryogenic SiGe Low-Noise Amplifiers
}

\author{
Sander Weinreb, Life Fellow, IEEE, Joseph C. Bardin, Student Member, IEEE, and Hamdi Mani
}

\begin{abstract}
This paper describes a method for designing cryogenic silicon-germanium (SiGe) transistor low-noise amplifiers and reports record microwave noise temperature, i.e., $2 \mathrm{~K}$, measured at the module connector interface with a $50-\Omega$ generator. A theory for the relevant noise sources in the transistor is derived from first principles to give the minimum possible noise temperature and optimum generator impedance in terms of dc measured current gain and transconductance. These measured dc quantities are then reported for an IBM SiGe BiCMOS-8HP transistor at temperatures from 295 to $15 \mathrm{~K}$. The measured and modeled noise and gain for both a single- and two-transistor cascode amplifier in the $0.2-3-G H z$ range are then presented. The noise model is then combined with the transistor equivalent-circuit elements in a circuit simulator and the noise in the frequency range up to $20 \mathrm{GHz}$ is compared with that of a typical InP HEMT.
\end{abstract}

Index Terms-Cascode, cryogenic, low-noise amplifier (LNA), noise parameters, silicon-germanium (SiGe).

\section{INTRODUCTION}

$\mathbf{R}$ EMARKABLE cryogenic performance in terms of cutoff frequency $(>500 \mathrm{GHz})$ and noise figure $(\mathrm{NF})(0.3 \mathrm{~dB}$ at $14 \mathrm{GHz}$ ) have recently been reported for silicon-germanium (SiGe) HBTs [1], [2]. The device physics leading to these results is documented in [3] and excellent reviews of the technology with hundreds of references are in a recent special issue of the PROCEEDING OF THE IEEE [4]. The focus of this paper is low-noise amplifiers (LNAs) for very low noise $(<0.1-\mathrm{dB} \mathrm{NF})$ at frequencies under $20 \mathrm{GHz}$ for applications where the noise in the generator (usually an antenna looking up) is well below $300 \mathrm{~K}$. These applications are radio astronomy, space communications, low-temperature physics research, and IF amplifiers for cryogenic mixers operating above $100 \mathrm{GHz}$.

Design of LNAs requires a knowledge of a set of four noise parameters of the transistor ( $T \mathrm{~min}, Z \mathrm{opt}$, and $R n$ are one set) and also the small-signal equivalent-circuit model. For the amplifiers and transistors under consideration, the following three problems arise.

1) For very low noise transistors, it is difficult to measure the $\mathrm{NF}$ (and, hence, noise parameters) with an accuracy better than $\pm 0.1 \mathrm{~dB}$ due to uncertainty in the noise generator and losses in the noise parameter measuring equipment. Thus, the error is of the order of the NF we wish to measure.

\footnotetext{
Manuscript received May 10, 2007; revised August 6, 2007. This work was supported by the Director's Fund of the Jet Propulsion Laboratory, California Institute of Technology, under a contract with the National Aeronautics and Space Administration.

The authors are with the Department of Electrical Engineering, California Institute of Technology, Pasadena, CA 91125 USA (e-mail: sweinreb@ caltech. edu).

Color versions of one or more of the figures in this paper are available online at http://ieeexplore.iee.org.

Digital Object Identifier 10.1109/TMTT.2007.907729
}

2) For SiGe HBTs, there are wide ranges of size and collector current to be considered and basic parameters such as current gain and transconductance vary widely with temperature and collector current. Thus, it is difficult to select an optimum device size, collector current, and embedding circuit.

3) The transistor small-signal equivalent circuit is usually known at room temperature, but not at cryogenic temperatures.

Our approach is to determine the transistor noise parameters from dc measurements, which are easily performed even at cryogenic temperatures. The operating temperatures, which are relevant and used for the measurements we report are: 1) $200 \mathrm{~K}$ for consideration of thermoelectric cooling; 2) 80 and $40 \mathrm{~K}$ for which small very long-life cryocoolers are available; and 3) $15 \mathrm{~K}$, which is currently widely used with field-proven closed-cycle cryocoolers. ${ }^{1}$

An outline of this paper is as follows.

1) Derive from first principles a simple noise model, which only contains the noise mechanisms that dominate the cryogenic noise performance in the low microwave frequency range. This has been done many times in the past, but is repeated to reveal the underlying assumptions and to assure correctness for temperatures much different than $300 \mathrm{~K}$.

2) The above low-frequency noise model requires only the knowledge of the current gain and transconductance as a function of collector current. Measurements of these quantities will be reported for SiGe transistors from the IBM SiGe BiCMOS-8HP 0.12- $\mu \mathrm{m}$ process.

3) Using 1) and 2), the noise parameters are determined as a function of collector current $I_{C}$ at the temperatures of interest.

4) The models will be validated by measurement of the noise temperature and gain of a transistor in a $50-\Omega$ system, $T 50$ and $S 21$, as a function of collector current and temperature.

5) The model will be extended by combining it with the smallsignal equivalent circuit, especially the internal HBT capacitances $\mathrm{Cbe}$ and $\mathrm{Cbc}$ to determine the noise parameters as a function of frequency. Tmin and $Z$ opt in the $0-20-\mathrm{GHz}$ range will be compared with values for a typical InP HEMT.

6) A cascode amplifier operating from 0.5 to $3 \mathrm{GHz}$ will be designed and measured as a function of frequency.

\section{Simple Low-FreQuency CRYogenic NoISE Model}

A low-frequency noise model that shows the dominant sources of noise is shown in Fig. 1. Here, $i_{\mathrm{BN}}$ and $i_{\mathrm{CN}}$ are the shot noise generators due to the $\mathrm{dc}$ base and collector

${ }^{1}$ Cryodyne model 350 cryocooler, CTI Cryogenics Div., Brooks Automation, Mansfield, MA. 


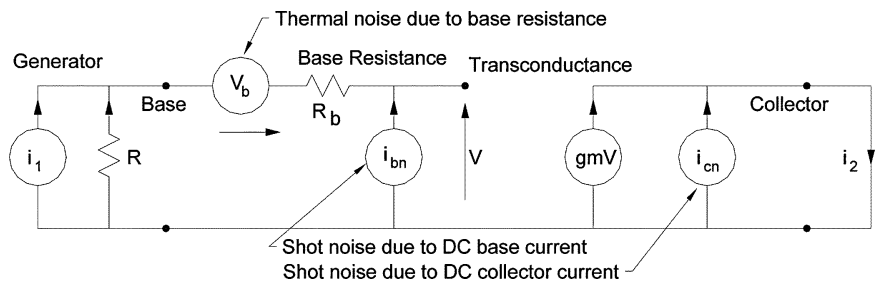

Fig. 1. Simple noise equivalent circuit of transistor.

bias currents. The correlation of these shot noise currents is controversial (see [5] and references therein), but our belief is that this correlation is negligible and that previous correlation measurements of input and output noise currents have been clouded by the correlation of terminal short circuit currents due to $g m$ and $R b$.

The noise temperature $T n$ of the transistor as a function of generator resistance $R$ is equal to the temperature of $R$, which will produce the same thermal noise as all the noise sources in the transistor. We thus equate the output mean-square shortcircuit current noise $i_{2 G}^{2}$ of the generator thermal noise to the same quantity due to the internal noise sources $i_{2 N}^{2}$

$$
\begin{aligned}
& i_{2 G}^{2}=i_{1}^{2} R^{2} g m^{2} \\
& i_{2 N}^{2}=i_{\mathrm{CN}}^{2}+g m^{2} v_{b}^{2}+g m^{2} R^{2} i_{\mathrm{BN}}^{2} .
\end{aligned}
$$

$T n$ can be determined in terms of useful parameters by equating (1) and (2) and using the shot noise relation $i_{n}^{2}=2 q I$, where $I$ is either the dc base current $I_{B}$ or collector current $I_{C}$, the dc current gain $\beta=I_{C} / I_{B}$, and the thermal noise relations $V_{b}^{2}=4 k T_{A} R_{b}$ and $i_{1}^{2}=4 k T n / R$. The equation for $T n$ is more easily understood if the fundamental constants $k$ and $q$ are expressed in convenient units $V o=k T o / q=0.025 \mathrm{~V}$ and $T o=290 \mathrm{~K}$ (independent of ambient temperature $T_{A}$ )

$$
\frac{T_{n}}{T_{o}}=\frac{T_{A}}{T_{o}} \frac{R_{b}}{R}+\frac{R I_{C}}{2 V_{o} \beta}+\frac{R I_{C}}{2 V_{o}} \frac{1}{R^{2} g m^{2}} .
$$

The terms are thus, from left to right, the contributions of base resistance thermal noise, base current shot noise, and the collector current shot noise. There is an optimum value of $R$, given below, which minimizes the noise, and this can be inserted in (3) to give $T \mathrm{~min}$, the noise minimum with respect to $R$

$$
R_{\mathrm{OPT}}=\left[\frac{\beta}{g m^{2}}+\beta \frac{T_{A}}{T_{o}} \frac{2 V_{o} R_{B}}{I_{C}}\right]^{1 / 2} .
$$

In the case where the thermal noise of $R_{B}$ is negligible, which is especially true for $T_{A} \ll 290 \mathrm{~K}, R_{\mathrm{OPT}}=\sqrt{ } \beta / \mathrm{gm}$. The minimum noise $T_{\mathrm{MIN}}$, which can be achieved by the transistor in any circuit configuration ${ }^{2}$ can be obtained by inserting (4) into (3). A good approximate value of $T_{\mathrm{MIN}}$, valid for negligible contribution from $R_{B}$, is reached when the contribution from the base shot noise, proportional to $R$ is equal to the contribution from collector shot noise proportional to $1 / R$. In this case,

$$
\frac{T_{\mathrm{MIN}}}{T_{O}} \cong \frac{I_{C} / V_{O}}{g m \sqrt{\beta}}
$$

${ }^{2}$ Lossless feedback can reduce the noise temperature of any amplifier to zero at the expense of power gain, but the noise temperature of cascaded stages, as expressed by noise measure, is invariant to lossless feedback [6]. which clearly shows the figure of merit $I_{C} /(g m \sqrt{ } \beta)$ for minimum noise.

The selection of optimum $I_{C}$ is much more difficult since $\beta$ is a strong function of $I_{C}$ at cryogenic temperatures and $g m$ is not quite linear with $I_{C}$. Our procedure will be to measure $\beta$ and $g m$ as functions of $I_{C}$ at the temperature of interest and then determine $T n$ and Ropt using (3) and (4).

The variation of $T n$ with scaling $N$ of transistor size can also be determined from the measured dc data for one transistor by considering $N$ transistors in parallel, but operated at a total collector current $I_{C}$. The measured single transistor $\beta$ and $g m$ at $I_{C} / N$ are then used to determine the $\beta$ and $g m$ of the combination. The $\beta$ for the combination is then the same (since both $I_{B}$ and $I_{C}$ are both multiplied by $N$ ) and $g m$ is given by $N$ times the $g m$ measured at $I_{C} / N$. Thus, if $g m$ was a linear function of $I_{C}$ then $g m$ would be invariant to $N$ for a given total $I_{C}$. For example if $N=10$, evaluate $\beta$ at the value measured at $I_{C} / 10$ and determine $g m$ as 10 times the $g m$ measured at $I_{C} / 10$.

From consideration of the $N$ parallel transistor case, Ropt is proportional to $1 / N$. From the measured data to follow, it will be shown that large transistors are desirable to bring Ropt close to $50 \Omega$ with $I_{C}$ in a current density range for high cryogenic $\beta$. However, large transistors have high capacitance and a compromise dependant upon desired frequency and bandwidth is required.

\section{MEASUREMENTS OF $\beta$ AND $g m$ AS FUNCTIONS OF $I_{C}$ AND TEMPERATURE}

A test strip of an IBM 8HP wafer processed in late 2006 was mounted with conductive epoxy in a coaxial test fixture, as shown in Fig. 2, and a $3 \times 18 \times 0.12 \mu \mathrm{m} \mathrm{HBT}$ was bonded to coplanar waveguide (CPW) lines leading to subminiature (SMA) connectors and dc-bias tees. Two Keithley 2400 source meters were used in a computer-controlled setup to scan $I b$ and $V$ ce, while measuring $V$ be and $I_{C}$.

Raw data showing $I_{C}$ versus $V$ be at six temperatures and $I_{C}$ versus $V$ ce curves at $15 \mathrm{~K}$ are shown in the Appendix. The main attributes of the $V$ be curve are the increase of threshold voltage from approximately $0.8 \mathrm{~V}$ at $293 \mathrm{~K}$ to $1.05 \mathrm{~V}$ for temperatures below $40 \mathrm{~K}$ and an increase in slope $(\mathrm{gm})$ with cooling. The $V$ ce curves show little change in $\beta$ with $V$ ce in the range of $0.2-1.2 \mathrm{~V}$.

The most important experimental results of this paper are summarized in Figs. 3 and 4, which show $\beta$ and $g m$ as a function of $I_{C}$ and temperature. The increase in $\beta$ and $g m$ by cooling from 293 to $40 \mathrm{~K}$ or less are remarkable. $\beta$ increases by a factor of $20(400-8000)$ and $g m$ increases by $3.2(10.1 \mathrm{~dB})$. Applied in (5), these factors reduce $T \mathrm{~min}$ at a given $I_{C}$ by a factor of 14.3

\section{LOW-FREQUENCY CRYOGENIC NOISE AND GAIN MEASUREMENTS}

It is important to note that the internal shot and thermal noise current sources are independent of frequency, although the noise currents reaching the device terminals are dependent upon frequency due to internal parasitic reactances. Thus, we can determine the noise sources at low frequencies where the parasitic effects are negligible and then determine the higher frequency 

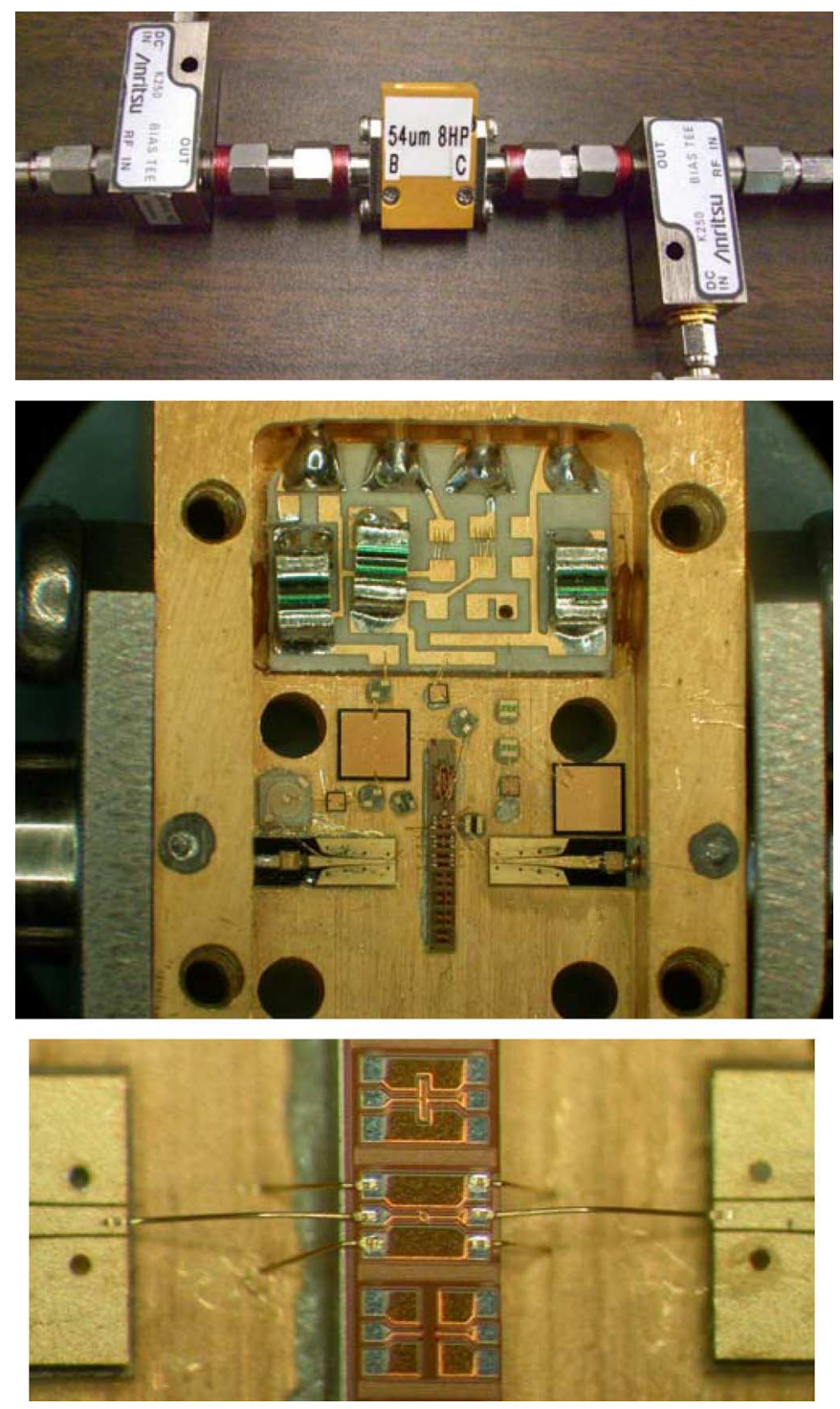

Fig. 2. (top) Coaxial test fixture with external dc-bias tee. (middle) Wafer segment bonded between $50-\Omega$ lines. (bottom) Test transistor with bonds to base input, collector output, and emitter to ground.

effects in Section V by incorporating the device small-signal model determined from $S$-parameters.

The coaxial test fixture shown in Fig. 2 was modified to facilitate noise measurements by installing an internal input dc block (22-pF coupling capacitor and $50-\mathrm{K}$ gate bias resistor) and bonding to an $180-\mu \mathrm{m}$ transistor $\left(10 \times 18 \times 0.12 \mu \mathrm{m}^{2}\right)$, which has $R$ opt closer to $50 \Omega$ than the $54-\mu$ m device $(3 \times 18 \times$ $0.12 \mu \mathrm{m}^{2}$ ) used for dc characterization.

The test configuration shown in Fig. 5 was used for the cryogenic noise and gain measurements at frequencies in the $0.2-1.2-\mathrm{GHz}$ range where both noise and gain have little variation with frequency The lower frequency is determined by the 22-pF coupling capacitor and the upper frequency by capacitance within the transistor, which is to be included in Section V. A cryocooler was used to cool the amplifier and an input $20-\mathrm{dB}$ attenuator to $15 \mathrm{~K}$ with higher temperatures available by applying power to a heating resistor affixed to the cold station. The input attenuator terminates the amplifier

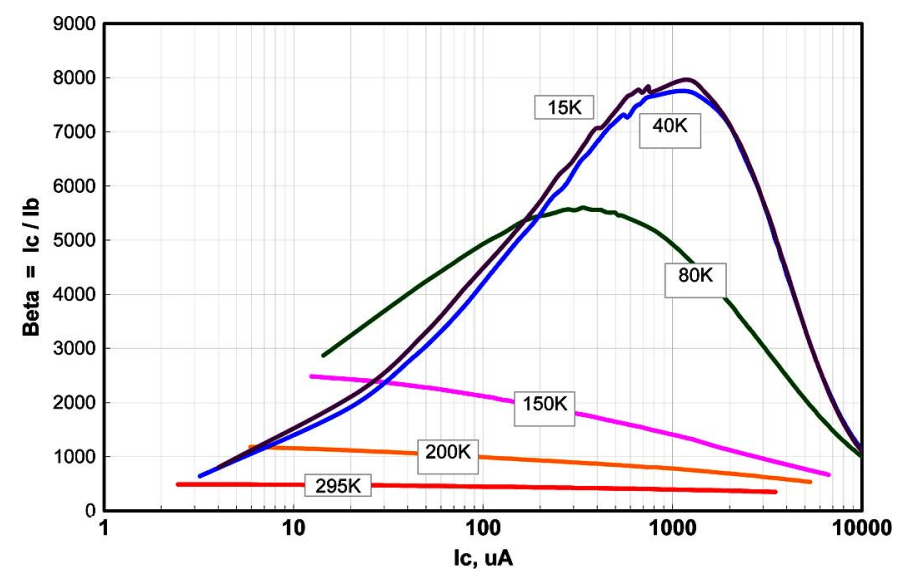

Fig. 3. $\beta=I_{C} / I b$ as a function of collector current at six temperatures for an IBM 8-HP 54- $\mu \mathrm{m} \mathrm{SiGe} \mathrm{transistor.} \mathrm{The} \mathrm{peak} \mathrm{cryogenic} \beta$ at $I c=1000 \mu \mathrm{A}$ thus occurs at a current density of $0.15 \mathrm{~mA} / \mu \mathrm{m}^{2}$, which is well below the current density for peak $g m$ or $F t . \beta$ is a function of current density and, thus, for example, a $10 \times 18 \times 0.12-\mu \mathrm{m}^{2}$ device would have peak $\beta$ for temperatures $<40 \mathrm{~K}$ at a collector current of $3.3 \mathrm{~mA}$.

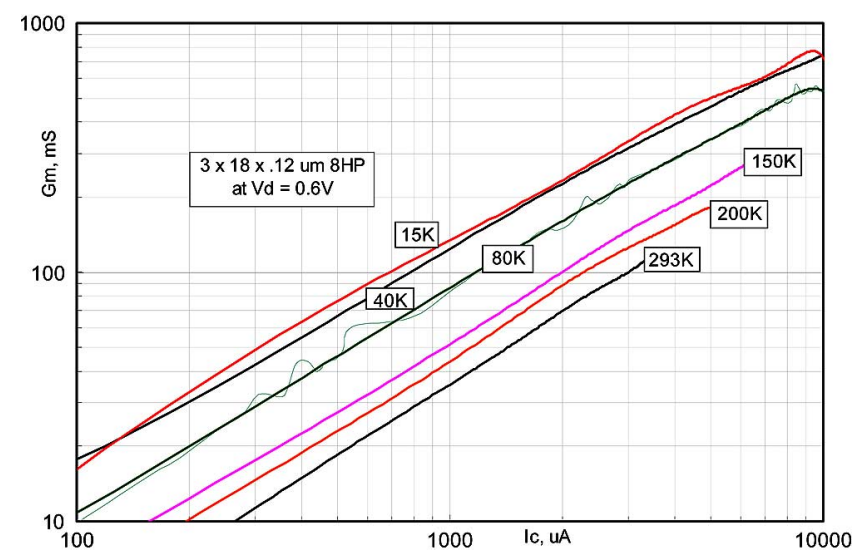

Fig. 4. Measured transconductance $g m$ as a function of collector current and temperature for an IBM $8 \mathrm{HP} 54-\mu \mathrm{m}$ device. This data has been measured at $\mathrm{dc}$ as $\partial I c / \partial V$ be and has been smoothed to remove measurement errors in the small increments. (Unsmoothed data is shown as dotted around the 80-K curve.) As an example of applying these curves, an $180-\mu \mathrm{m}$ device at $15 \mathrm{~K}$ would have a $g m$ of $1.02 \mathrm{~S}$ at $10 \mathrm{~mA} I_{C}$, which is 3.3 times the value on the above curve at $3 \mathrm{~mA}$.

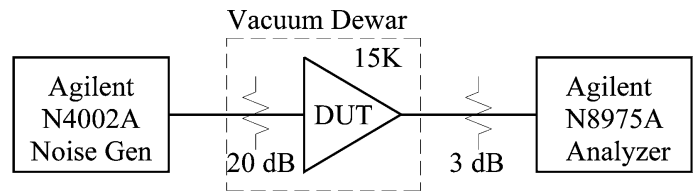

Fig. 5. Cryogenic noise test configuration. The cooled 20-dB attenuator at input of the device-under-test provides a calibrated low-temperature input termination and greatly increases the accuracy of low-noise measurements.

in a generator with $18-\mathrm{K}$ noise (rather than $300 \mathrm{~K}$ without the attenuator) and greatly increases the accuracy of the cryogenic noise measurement. External to the vacuum chamber, an Agilent N4002A, 12-17-dB ENR "smart" noise source, and N8975A noise analyzer are utilized. This test set has been used for the noise measurement of over a $200 \mathrm{InP}$ cryogenic LNA and comparison of the noise data with the National Institute of Standards and Technology (NIST) and other laboratories has indicated an accuracy of $\pm 1 \mathrm{~K}$ for frequencies up to $10 \mathrm{GHz}$. 
TABLE I

NOISE TEMPERATURE OF $180-\mu \mathrm{m}$ 8-HP TRANSISTOR ALL AT $V \mathrm{ce}=0.6 \mathrm{~V}, I_{C} 6-11 \mathrm{~mA}$, AVERAGED $0.2-1.2 \mathrm{GHz}$

\begin{tabular}{|c|c|c|r|r|r|}
\hline \multirow{2}{*}{ Quantity } & \multicolumn{5}{|c|}{ Ambient Temperature, K } \\
\cline { 2 - 6 } & $\mathbf{2 9 3}$ & $\mathbf{2 0 0}$ & $\mathbf{8 0}$ & $\mathbf{4 0}$ & $\mathbf{1 5}$ \\
\hline T50, measured, K & 46.0 & 28.0 & 6.1 & 2.0 & 2.2 \\
\hline T50, at transistor, K & 26.0 & 17.0 & 4.5 & 1.7 & 2.1 \\
\hline T50, modeled, K & 23.5 & 15 & 4.7 & 2.5 & 1.8 \\
\hline Tmin, modeled, K & 22.9 & 13 & 3.6 & 1.1 & 0.9 \\
\hline S21 measured, dB & 23.9 & 27.9 & 32.0 & 32.5 & 32.8 \\
\hline
\end{tabular}

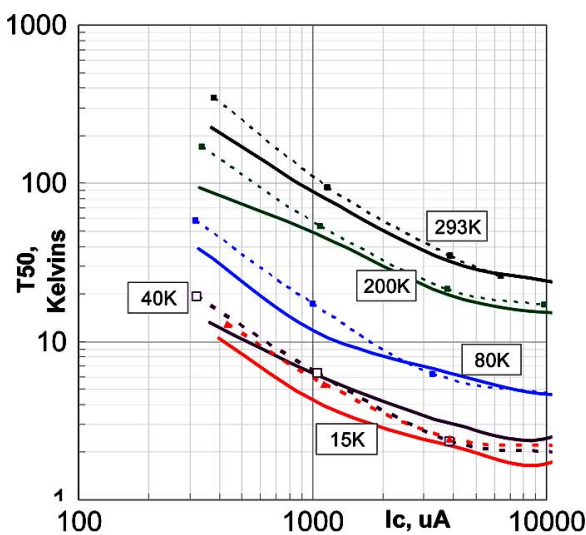

Fig. 6. Measured (dashed line) and modeled noise at 50- $\Omega$ generator impedance as a function of collector current and temperature for $180-\mu \mathrm{m} \mathrm{IBM}$ 8-HP SiGe HBT. The noise temperature is averaged from 0.2 to $1.2 \mathrm{GHz}$ with small deviations from the average in that frequency range.

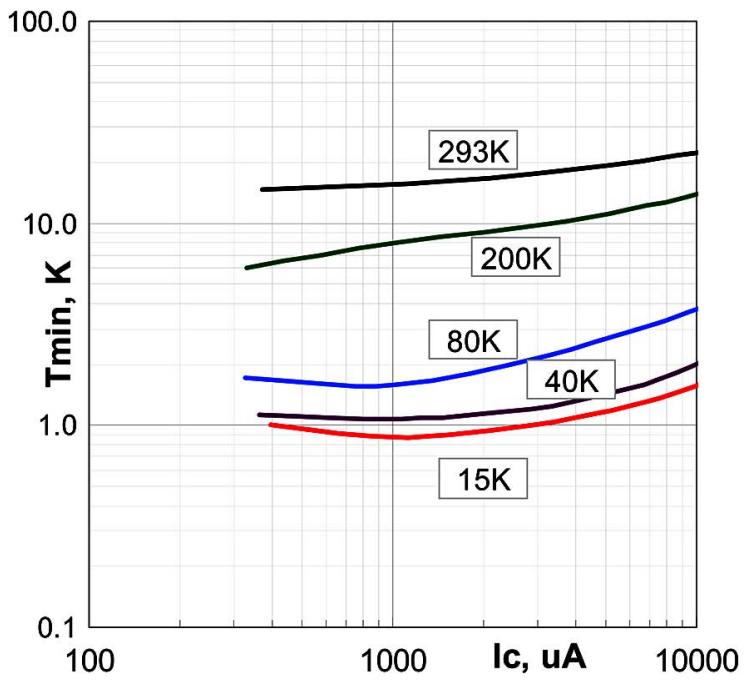

Fig. 7. Modeled minimum noise $T \mathrm{~min}$ achievable with optimum generator impedance $R$ opt for an $180-\mu \mathrm{m}$ IBM 8 -HP HBT.

A summary of the noise results is given in Table I with more details in Figs. 6-9.

The noise temperature results are outstanding. To the best of our knowledge, the module noise is within $\pm 1 \mathrm{~K}$ of the lowest yet reported for a microwave amplifier. The results at $15 \mathrm{~K}$ are at a dc power of $0.6 \mathrm{~V} \times 7.1 \mathrm{~mA}=4.2 \mathrm{~mW}$. However, at $0.15 \mathrm{~V} \times 3.3 \mathrm{~mA}=0.5 \mathrm{~mW}$, the noise only increased by $0.7 \mathrm{~K}$ and gain decreased by $3.2 \mathrm{~dB}$. A tradeoff of power consumption

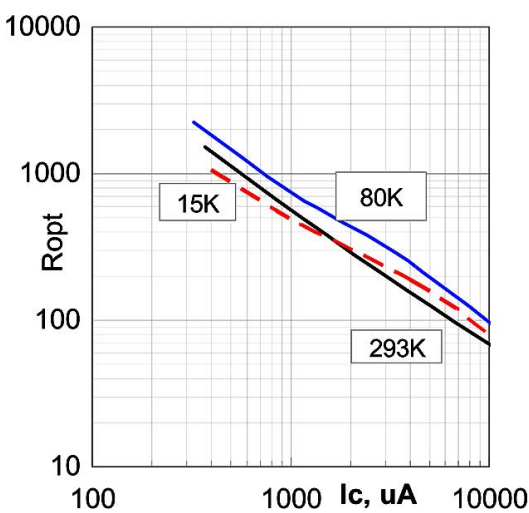

Fig. 8. Modeled low-frequency Ropt as a function of collector current at three temperatures for a $180-\mu \mathrm{m}$ device. There is little variation with temperature because the ratio of $\sqrt{ } \beta$ and $g m$ in (4) do not vary by a large amount with temperature. The frequency variation of $Z$ opt at $15 \mathrm{~K}$ is shown in Fig. 11

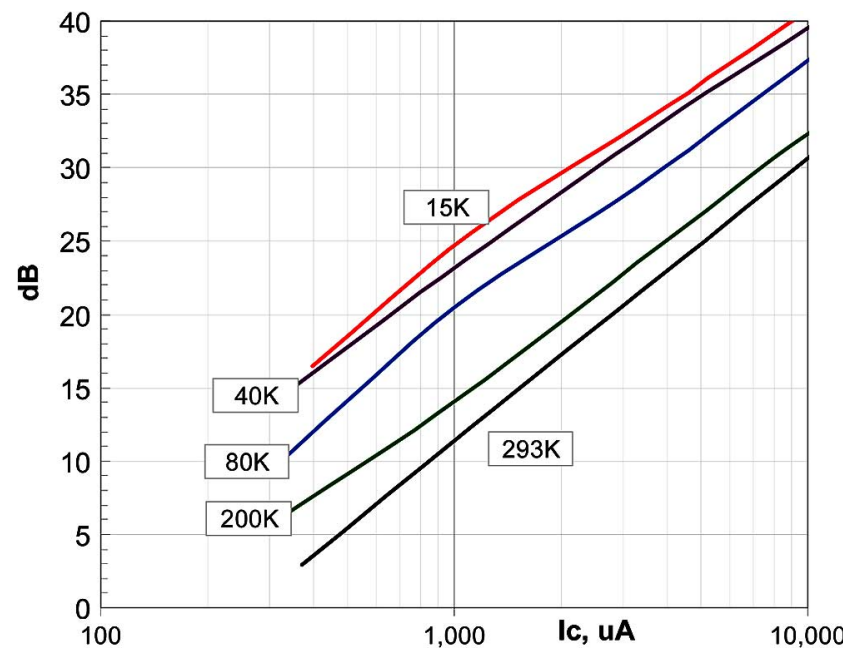

Fig. 9. Modeled low-frequency $S 21$ versus $I_{C}$ and temperature for the $180-\mu \mathrm{m}$ HBT.

versus gain compression power is thus available and is important for large arrays of cryogenic detectors or mixers.

The top row in Table I is the measured noise at the module SMA input connector. The second row is the noise referred to the transistor input pads and corrects for the loss of the connector, input transmission line, coupling capacitor, bond wires, and lines from the bond pads to the transistor. At $293 \mathrm{~K}$ this loss is estimated to be $0.27 \mathrm{~dB}$, and this value is scaled down with temperature proportional to the microwave surface resistance, which goes as the root of the dc resistivity of copper as a function of temperature. At $40 \mathrm{~K}$ and below, the loss correction is negligible.

\section{Noise Versus FreQuency Model}

As a basis for future amplifier designs, a size-scalable smallsignal noise model of an 8-HP HBT was analyzed in a circuit simulator [7]. The equivalent-circuit elements were determined from 300-K IBM model data with the addition of $\beta$ and $\mathrm{gm}$ as a function of $I_{C}$ and temperature from the measurements summarized in Figs. 3 and 4. Thus, the assumption is that the only equivalent-circuit elements that vary with temperature are $\beta$ and $g m$. (Previous cryogenic $S$-parameter measurements [2] 


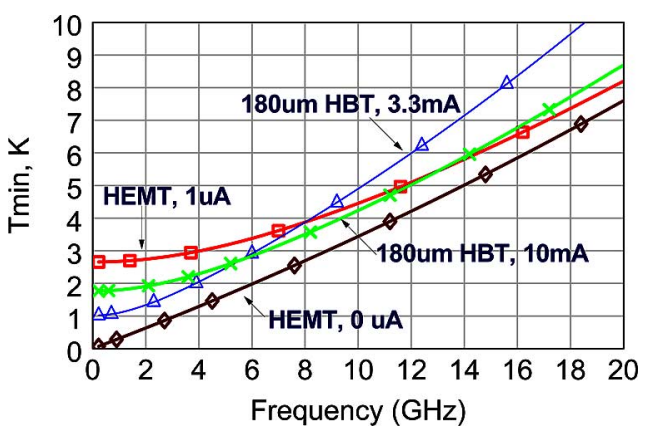

Fig. 10. Modeled minimum noise temperature as a function of frequency at $15 \mathrm{~K}$ for two HBTs and two InP HEMTs shown in Table II. The noise of the $54-\mu \mathrm{m} \mathrm{HBT}$ at $I c=3 \mathrm{~mA}$ shown in column 1 of Table II is identical to that of the $180-\mu \mathrm{m} \mathrm{HBT}$ at $I c=10 \mathrm{~mA}$ The voltage and current per transistor are similar for InP and SiGe (around $0.8 \mathrm{~V}$ and $10 \mathrm{~mA}=8 \mathrm{~mW}$ ) and can be optimized if necessary for lower power at little increase in noise.

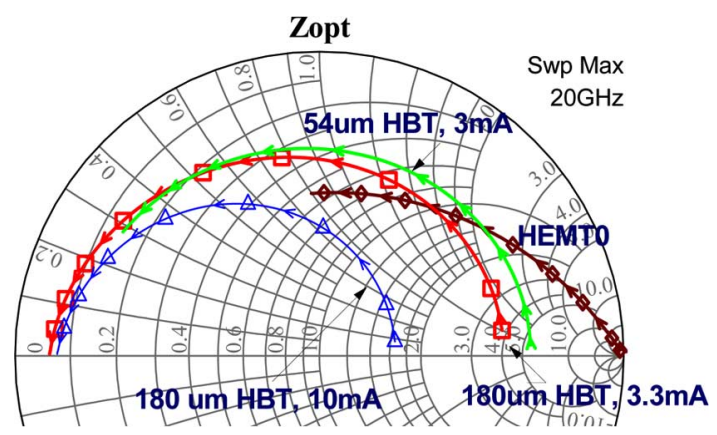

Fig. 11. Modeled optimum noise impedance $Z$ opt, $0.2-20 \mathrm{GHz}$, at $15 \mathrm{~K}$ for the cases shown in Table II. Note that if this impedance was expressed as an admittance, i.e., $Y$ opt, then Gopt is more constant with frequency than Ropt.

TABLE II

COMPARISON OF SiGe HBT AND HEMT AT $15 \mathrm{~K}$

\begin{tabular}{|c|c|c|c|c|c|}
\hline Case & $\mathbf{1}$ & $\mathbf{3}$ & $\mathbf{4}$ & HEMT0 & HEMT1 \\
\hline Width, um & 54 & 180 & 180 & 200 & 200 \\
\hline Ic, mA & 3 & 10 & 3.3 & 6 & 6 \\
\hline Beta & 5600 & 5600 & 7800 & - & - \\
\hline gm, mS & 300 & 1000 & 470 & 220 & 220 \\
\hline Tmin, $1 \mathrm{GHz}$ & 1.8 & 1.8 & 1.1 & 0.4 & 2.7 \\
\hline Tmin, $8 \mathrm{GHz}$ & 3.5 & 3.5 & 3.9 & 2.7 & 3.9 \\
\hline
\end{tabular}

have shown that the base resistance $R b$ is lowered by a factor of 2 with cooling and this would improve the noise at higher frequencies.)

The circuit simulator was used to compute $T$ min, the minimum noise temperature with respect to generator impedance, as well as the optimum value of this impedance $Z$ opt; these results are shown in Figs. 10 and 11. The values, at $15 \mathrm{~K}$, for three different cases of HBT size and $I_{C}$, are also tabulated in Table II.

For comparison with these results, $0.1-\mu \mathrm{m}$ InP HEMT transistors previously measured at $15 \mathrm{~K} \mathrm{[8],} \mathrm{[9]} \mathrm{have} \mathrm{been} \mathrm{included.}$ The HEMT noise at low microwave frequencies depends upon the gate leakage current ranging from $<0.1 \mu \mathrm{A}$ (denoted as $0 \mu \mathrm{A}$ in Fig. 10) for selected transistors to $1 \mu \mathrm{A}$ for a mediocre transistor. The results show comparable noise temperatures. Improved devices of both types are expected with shorter gate $(0.035 \mu \mathrm{m})$ HEMT devices now available and expectations of

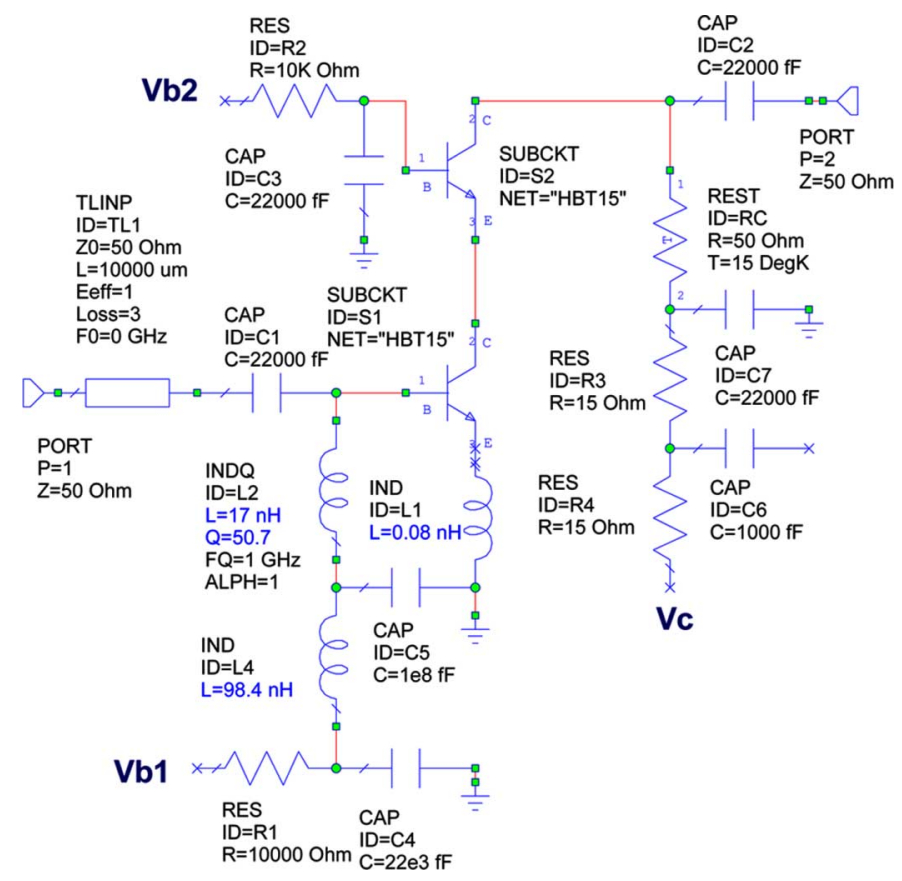

Fig. 12. Schematic of cascode $0.5-3-\mathrm{GHz}$ amplifier modeled with circuit simulation software [7] and implemented as shown in Fig. 13 with bond-wire connections of small MIC components.

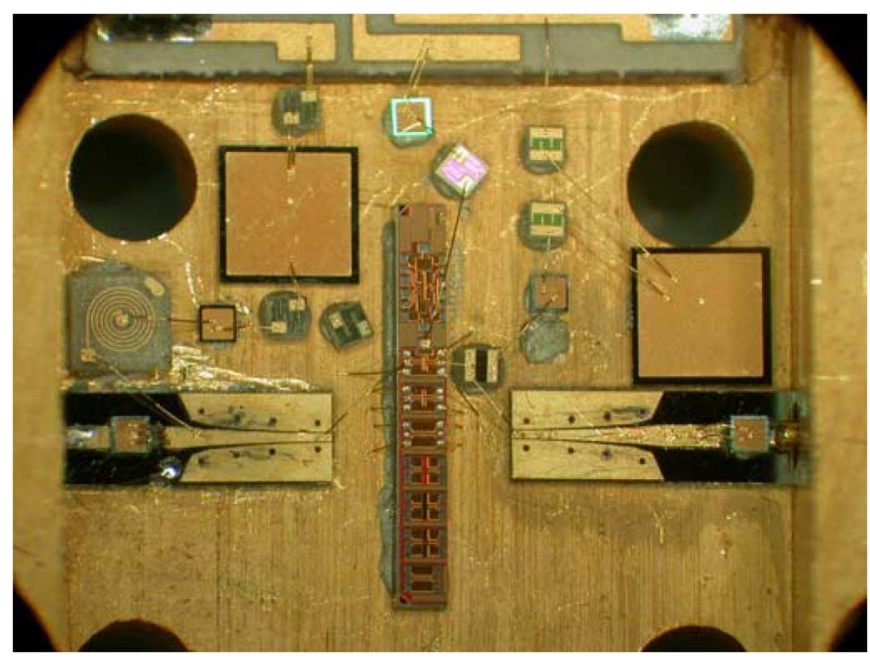

Fig. 13. Cascode amplifier implemented in a SMA connector module with internal dc blocks. The cascode of two transistors is within one 500- $\mu \mathrm{m}$ square of the larger wafer strip containing other test circuits. An input spiral inductor, $L 2$ on the schematic, is shown on the left and the collector load resistor $R C$ is to the right of the chip.

further improvement of SiGe HBTs. Note that the $Z$ opt reflection coefficient angle change with frequency for the HEMT device is approximately $1 / 2$ that of the HBTs, and this will result in greater noise bandwidth. On the other hand, the HBTs have higher yield and offer the opportunity for large-scale integration.

\section{CASCode AmPlifier EXAMPLE}

In order to further validate the design methods and demonstrate a simple extreme LNA. A single-stage cascode microwave integrated circuit (MIC) amplifier was assembled, as shown in Figs. 12 and 13, with measured and modeled results shown in 
TABLE III

MEASURED AMPLIFIER RESULTS AT 300 AND $15 \mathrm{~K}$

\begin{tabular}{|c|c|c|}
\hline Physical Temperature, $\mathrm{K}$ & 300 & 15 \\
\hline Noise temperature, $\mathrm{K}$, at $1 \mathrm{GHz}$ & 60 & 2.1 \\
\hline Gain, dB, at $1 \mathrm{GHz}$ & 22 & 28 \\
\hline Input return loss, dB, 0.5 to $5 \mathrm{GHz}$ & $>2$ & $\mathrm{NM}$ \\
\hline Output return loss, dB, 0.6 to $3 \mathrm{GHz}$ & $>12$ & $>12$ \\
\hline
\end{tabular}

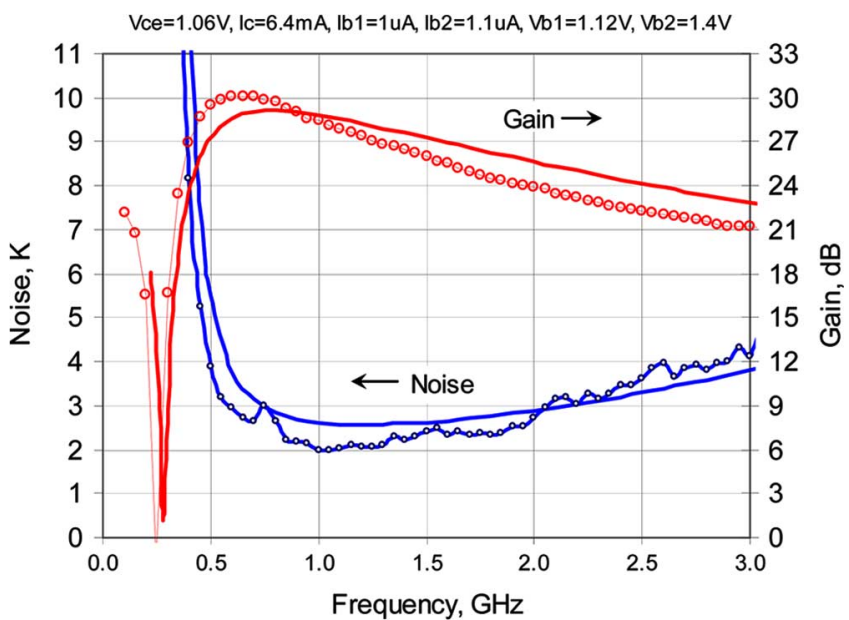

Fig. 14. Measured and modeled noise and gain of a single-stage cascode SiGe IBM 8-HP $180-\mu \mathrm{m}$ transistor at $15 \mathrm{~K}$. We believe the large dip in gain at $0.25 \mathrm{GHz}$ is due a resonance between two capacitors on the gate bias line and can be easily remedied.

Table III and Fig. 14. The noise is measured at an input SMA connector with no corrections for fixture loss. The shape of $S 21$ and $S 22$ did not change appreciably from 300 to $15 \mathrm{~K}$, indicating that the reactive elements in the device equivalent circuit do not change by large amounts and confirming that the cryogenic dc measurements of $\beta$ and $g m$ in addition to the $300-\mathrm{K}$ model are sufficient for cryogenic design.

This example amplifier was constructed with long bond-wire connections between existing undiced discrete transistor chips and is by no means optimum with respect to input return loss or gain flatness. The input return loss is poor due to the lack of feedback to improve input match. At frequencies above $6 \mathrm{GHz}$, the $S$-parameters vary widely and the output return loss is $<0 \mathrm{~dB}$ at $8 \mathrm{GHz}$; this is believed to be due to long bond-wire coupling and lack of proper layout. The 3-dB pad on the output, as shown in Fig. 5, assures stability and no oscillations were observed with a 26-GHz spectrum analyzer. A two-stage monolithic microwave integrated circuit (MMIC) amplifier removing these deficiencies has been designed and will be reported in a future publication.

\section{CONCLUSION}

We have demonstrated that SiGe bipolar transistors have excellent potential to become the device of choice for very lownoise cryogenic amplifiers required in radio astronomy, space communications, and other microwave applications where the generator noise is much less than $300 \mathrm{~K}$. The measured noise temperature in the $0.7-3-\mathrm{GHz}$ range and the modeled noise temperature to $20 \mathrm{GHz}$ are comparable to that measured with the best 0.1- $\mu \mathrm{m}$ InP HEMT transistors, yet SiGe has advantages of on-chip integration with CMOS, very high yield, a rich stable of accurate passive components, and a more rapid development
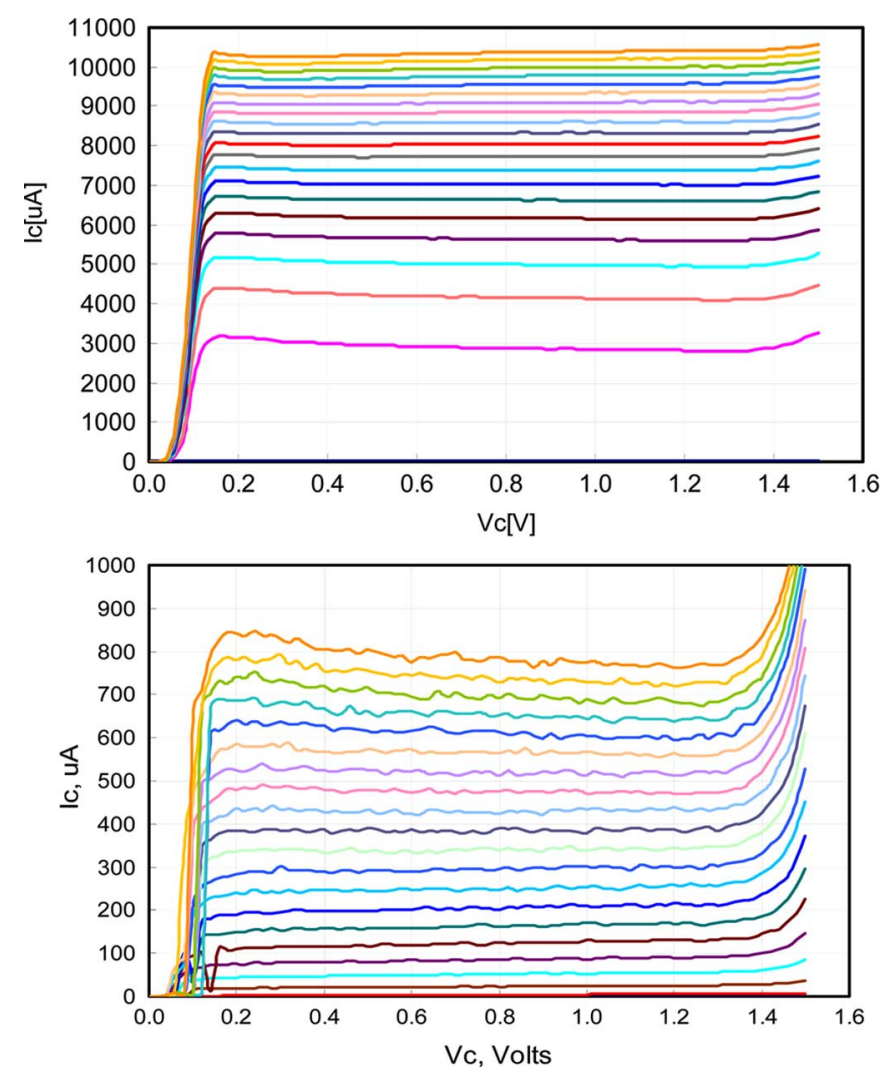

Fig. 15. $I_{C}$ versus $V_{c}$ at $15 \mathrm{~K}$. (top) For $0.5-\mu \mathrm{A}$ steps of $I b$. (bottom) For 5-nA steps.

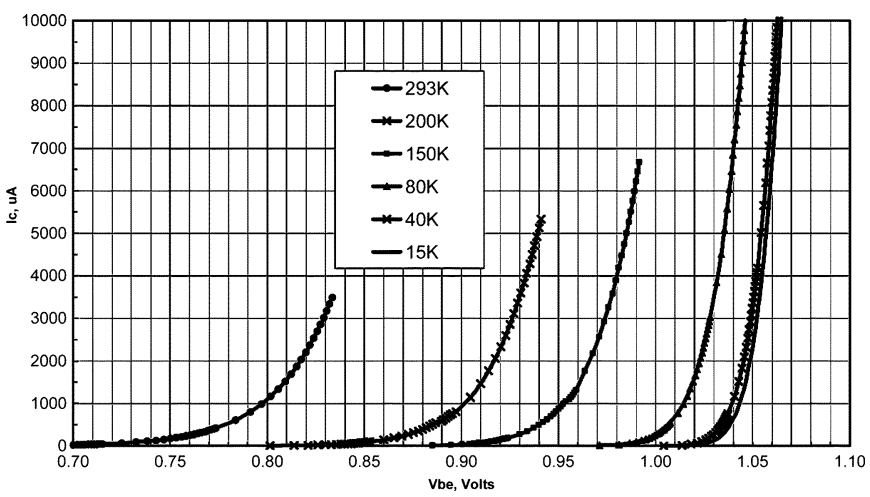

Fig. 16. $I_{C}$ as a function of $V$ be at six temperatures. The slope gives the transconductance $g m$.

pace. This paper has demonstrated the design procedure of using $\mathrm{dc}$ measurements to determine a noise model as a function of temperature and collector current. This de data in conjunction with a transistor small-signal model can then be used to determine the optimum device size, collector current, and generator impedance.

\section{APPENDIX}

Additional cryogenic dc data for $54-\mu \mathrm{m}$ HBT is shown in Figs. 15 and 16.

\section{ACKNOWLEDGMENT}

The authors appreciate the help of the Mixed Signal and RF Group, California Institute of Technology, Pasadena, particu- 
larly, Prof. A. Hajimiri and A. Babakhani for sample transistors and help with the IBM process. The authors thank Prof. D. Rutledge and G. Jones, both with the California Institute of Technology, for helpful discussions of the work and R. Gawande and G. Wang, both with the California Institute of Technology, for early studies and measurements of SiGe devices. The authors appreciate the help of N. Wadefalk, Chalmers University, Göteborg, Sweden, for contributions to the noise measurement calibration and amplifier testing and construction techniques. The tested transistors were processed by IBM under the Trusted Foundry Program and further processing of the authors' designs in this program is under the sponsorship of Dr. K. Johnson, U.S. Naval Observatory, Washington, DC.

\section{REFERENCES}

[1] R. Krthivasan, Y. Lu, J. D. Cressler, J.-S. Rieh, M. H. Khater, D. Ahlgren, and G. Freeman, "Half-terahertz operation of SiGe HBTs," IEEE Electron Device Lett., vol. 27, no. 7, pp. 567-569, Jul. 2006.

[2] B. Banerjee, S. Venkataraman, Y. Lu, Q. Liang, C.-H. Lee, S. Nuttinck, D. Heo, Y.-J. E. Chen, J. D. Cressler, J. Laskar, G. Freeman, and D. C. Ahlgren, "Cryogenic operation of third-generation, 200-GHz peak $f_{T}$ silicon-germanium heterojunction bipolar transistors," IEEE Trans. Electron Devices, vol. 52, no. 4, pp. 585-593, Apr. 2005.

[3] J. D. Cressler and G. Niu, Silicon-Germanium Heterojunction Bipolar Transistors. Norwood, MA: Artech House, 2003.

[4] R. Singe, D. Harame, and B. Myerson, Eds., Proc. IEEE (Special Issue), vol. 93, no. 9, Sep. 2005.

[5] M. A. Selim, "Accurate high-frequency noise modeling in SiGe HBTs," RF Design, pp. 24-32, Mar. 2006.

[6] H. A. Haus and R. B. Adler, "Optimum noise performance of linear amplifiers," Proc. IRE, vol. 46, no. 8, pp. 1517-1533, Aug. 1958.

[7] Microwave Office. ver. 6.53, Appl. Wave Res., El Segundo, CA.

[8] R. Grundbacher, R. Lai, M. Barsky, R. Tsai, T. Gaier, S. Weinreb, D. Dawson, J. J. Bautista, J. F. Davis, N. Erickson, T. Block, and A. Oki, "0.1- $\mu \mathrm{m}$ InP HEMT devices and MMICs for cryogenic low noise amplifiers from $X$-band to $W$-band," in 14th Indium Phosphide and Related Mater. Conf., May 2002, pp. 455-458.

[9] J. D. Pandian, L. Baker, G. Cortes, P. F. Goldsmith, A. A. Deshpande, R. Ganesan, J. Hagen, L. Locke, N. Wadefalk, and S. Weinreb, "Lownoise 6-8 GHz receiver," IEEE Micro, vol. 7, no. 6, pp. 74-84, Dec. 2006.

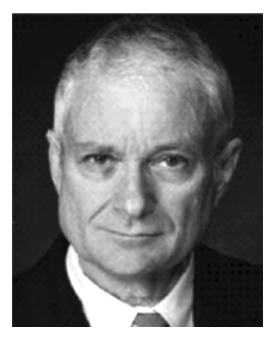

Sander Weinreb (S'56-M'63-SM'71-F'78-LF'02) was born in New York, NY, on December 9, 1936. He received the B.S. and Ph.D. degrees in electrical engineering from the Massachusetts Institute of Technology (MIT), Cambridge, in 1958 and 1963, respectively.

Since 1999, he has been a Faculty Associate with the California Institute of Technology, Pasadena, and a Principal Staff Member with the Jet Propulsion Laboratory, Pasadena, CA, where his major interest is large arrays for space communication and radio astronomy. He was Head of the Electronic Division (1965-1985) and Assistant Director (1986-1988) at the National Radio Astronomy Observatory, Charlottesville, VA. He has been a Visiting Researcher or faculty member with the University of California at Berkeley (1976-1978), University of Virginia (1987-1989), and University of Massachusetts at Amherst (1996-1999). From 1989 to 1996, he was Principal Scientist and Leader of the Millimeter Wave Design and Test Group, Martin Marietta Laboratories, Baltimore, MD. He has authored over 150 publications in the areas of digital correlation techniques.

Dr. Weinreb has served on many IEEE Microwave Theory and Techniques Society (IEEE MTT-S) Technical Committees and paper review teams. He was a 1985 IEEE MTT-S National Lecturer, giving 35 presentations of "Radio Astronomy - A Challenge to the Microwave Engineer." He has served on Advisory Committees for several national and international radio observatories, was an advisor to the National Aeronautics and Space Administration (NASA) Search for Extraterrestrial Life Program (1991-1997), and a U.S. delegate to the Soviet Space Research Institute (1986-1987). He was nominated to the U.S. Army Science Board (1991). He is a member of Commission J of URSI.

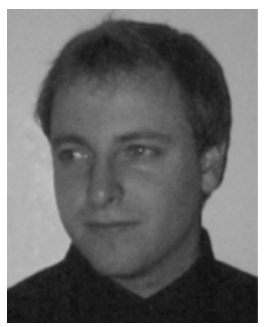

Joseph C. Bardin (S'01) received the B.S. degree in electrical engineering from the University of California at Santa Barbara, in 2003, the M.S. degree in electrical engineering from the University of California at Los Angeles (UCLA), in 2005, and is currently working toward the Ph.D. degree in electrical engineering at the California Institute of Technology, Pasadena.

In Summer 2001, he was with Titan, San Diego, CA, where he developed a flexible $L$-band synthesizer system, which was later integrated in a heterodyne remote-sensing system. From 2003 to 2007, he was with the Jet Propulsion Laboratory, Pasadena, CA, where he was involved with a satellite-link-based local oscillator distribution system for use with very long baseline interferometers, as well as the testing and development of the prototype receiver system for the NASA Deep Space Network Array. Since 2005, he has been a Research Assistant with the California Institute of Technology. His research interests include very wide bandwidth integrated circuit receivers, cryogenic circuits and applications, device modeling, and compact planar filters.

Mr. Bardin was the recipient of a summer undergraduate research fellowship with the California Institute of Technology in Summer 2002. He was also a recipient of a 2006 IEEE Microwave Theory and Techniques Society (IEEE MTT-S) Graduate Student Fellowship Award.

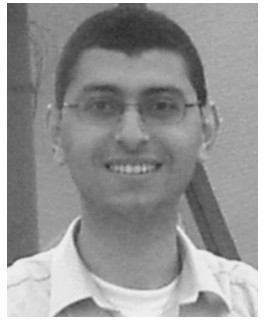

astronomy receivers.
Hamdi Mani was born in Hammam Sousse, Tunisia, on September 18, 1980.

After graduating from secondary school, he began his self-education in the areas of radio astronomy and microwave receivers. In 2005, he became a Research Technician with the Electrical Engineering Department, California Institute of Technology, Pasadena. $\mathrm{He}$ specializes in microassembly, computer-aided testing, measurement of MMIC cryogenically cooled very low-noise amplifiers, microwave network analyzer measurements, and the integration of radio

Mr. Mani is a member of the Society of Amateur Radio Astronomers (SARA). 